\title{
VISA AMONGST MRSA ISOLATES IN A TERTIARY CARE HOSPITAL IN MANIPUR
}

\author{
Urvashi Chongtham ${ }^{1}$, Sonita Mayengbam², Smeeta Huidrom³, Huidrom Lokhendro Singh ${ }^{4}$
}

${ }^{1}$ Associate Professor, Department of Microbiology, Jawaharlal Nehru Institute of Medical Sciences, Imphal, Manipur.

2 Postgraduate Student, Department of Microbiology, Jawaharlal Nehru Institute of Medical Sciences, Imphal, Manipur.

${ }^{3}$ Tutor, Department of Microbiology, Jawaharlal Nehru Institute of Medical Sciences, Imphal, Manipur.

${ }^{4}$ HOD, Department of Microbiology, Jawaharlal Nehru Institute of Medical Sciences, Imphal, Manipur.

\begin{abstract}
\section{BACKGROUND}

Methicillin-resistant Staphylococcus aureus (MRSA) is a major cause of hospital acquired and community-acquired infections. MRSA isolates are important for their resistance to many commonly used antibiotics. Vancomycin, a glycopeptide, was considered to be the best alternative for the treatment of MRSA. However, there are increasing number of reports indicating the emergence of vancomycin-resistant $S$. aureus (VRSA). The prevalence of MRSA in a similar setup in the state showed a prevalence of MRSA to be $84 \%$ in 2008. Therefore, the objective of this study was to find prevalence of the VISA amongst the MRSA isolates in our Hospital.
\end{abstract}

\section{MATERIALS AND METHODS}

A cross-sectional study was conducted in a tertiary care teaching hospital of Imphal, Manipur, India during the period from December 2015 to May 2016. A total of 126 Staphylococcus aureus isolates from different clinical specimens like wound swabs, pus and urine received from inpatients and outpatients of this referral tertiary care hospital were included in the study. Staphylococci were obtained either as pure culture or as an isolate of a polymicrobial infection. S. aureus was identified conventionally. MRSA were identified using cefoxitin disc $(30 \mu \mathrm{g})$ by Kirby-Bauer disc diffusion technique according Clinical and Laboratory Standards Institute (CLSI) guidelines 2014. All MRSA isolates were subjected to susceptibility testing by the Kirby-Bauer's disc diffusion method using different antimicrobial agents. All the MRSA isolates irrespective of their susceptibility pattern to Vancomycin by disc diffusion were resorted to E-test (bioMerieux) to determine the minimum inhibitory concentration (MIC). MIC $\leq 2 \mu \mathrm{g} / \mathrm{mL}$ were identified as sensitive, 4 to $8 \mathrm{mg} / \mathrm{L}$ were identified as vancomycin-intermediate and isolates with a vancomycin MIC $>16 \mathrm{mg} / \mathrm{L}$ were identified as vancomycin-resistant $S$. aureus according to CLSI, 2014. Quality control was performed using the S. aureus ATCC 25213 strain. Antibiotic susceptibility testing of VISA isolates was done by Kirby-Bauer's Disc diffusion method using the same drugs used for MRSA.

\section{RESULTS}

Prevalence of MRSA was (100/126) 79.36\%. MRSA isolates were $100 \%$ resistant to Penicillin followed by Ampicillin (90\%), Cotrimoxazole (76\%). 100\% sensitivity was seen with Linezolid followed by Ciprofloxacin (75\%), Ofloxacin (79\%), Nitrofurantoin (76\%), Amikacin (62\%), Gentamycin (70\%) and Tetracycline (66\%). Prevalence of VISA isolates was 3\% (3/100) in this study. Amongst the VISA isolates, linezolid was the most sensitive for all the three isolates and nitrofurantoin was sensitive for both the urine isolates and clindamycin was also sensitive for the only pus isolate.

\section{CONCLUSION}

This study emphasises the need to study the prevalence and antimicrobial susceptibility pattern of MRSA isolates area-wise in order to guide policy on the appropriate use of antibiotics which would minimise the irrational use of vancomycin and so the emergence of resistance to vancomycin.

\section{KEYWORDS}

Reduced Vancomycin Susceptibility, E-strip, Antibiogram.

HOW TO CITE THIS ARTICLE: Chongtham U, Mayengbam S, Huidrom S, et al. VISA amongst MRSA isolates in a tertiary care hospital in Manipur. J. Evolution Med. Dent. Sci. 2017;6(77):5498-5501, DOI: 10.14260/Jemds/2017/1193

\section{BACKGROUND}

Methicillin-resistant Staphylococcus aureus (MRSA) is among a major cause of hospital acquired and community-acquired infections. The methicillin resistance in MRSA is due to the acquisition of mecA gene, which encodes the low affinity penicillin-binding protein (PBP2a), a cell wall transpeptidase, which, in conjunction with native PBP2, allows continued cell

Financial or Other, Competing Interest: None.

Submission 19-08-2017, Peer Review 13-09-2017,

Acceptance 18-09-2017, Published 25-09-2017.

Corresponding Author:

Dr. Sonita Mayengbam,

Jawaharlal Nehru Institute of Medical Sciences,

Porompat, Imphal East-795005, Manipur.

E-mail: sonitadevi@yahoo.co.uk

DOI: $10.14260 /$ jemds $/ 2017 / 1193$

\section{(c) (i) $(-)$}

wall synthesis in the presence of $\beta$-lactams. ${ }^{1}$ MRSA isolates are important for their resistance to many commonly used antibiotics. They exhibit remarkable versatility in their behaviour towards antibiotics which poses a serious therapeutic problem. Thus, MRSA remains as a major cause of morbidity and mortality among hospitalised patients despite the availability of numerous effective anti-Staphylococcal antibiotics. ${ }^{2}$ Vancomycin, a glycopeptide, was considered to be the best alternative for the treatment of MRSA. However, there are increasing numbers of reports indicating the emergence of vancomycin-resistant $S$. aureus (VRSA). Initially vancomycinintermediate $S$. aureus (VISA) noted in Japan in 1996 and subsequently in United States in 1997.3 Sequential mutations in Vancomycin Susceptible Staphylococcus aureus (VSSA) led to the emergence of vancomycin heteroresistant-intermediate 
Staphylococcus aureus (hVISA) and ultimately Vancomycin intermediate resistance Staphylococcus aureus (VISA).

The hallmark changes are alterations in the bacterial cell wall resulting in reduced autolytic activity and wall thickening. Multiple genetic mutations have been implicated in the pathogenesis of these cell wall modifications. While the genetic determinants of hVISA and VISA are partially understood, the relative contributions of these mutations-and of altered cellular processes that contribute to hVISA and VISA-in determining the vancomycin MIC are unknown. ${ }^{4}$ The prevalence of MRSA in a similar set up in the state showed a prevalence of MRSA to be $84 \%$ in 2008.5 Therefore, the objective of this study was to find incidence of the VISA amongst the MRSA isolates in our Hospital.

\section{MATERIALS AND METHODS}

A cross-sectional study was conducted in a tertiary care teaching hospital of Imphal, Manipur, India during the period from December 2015 to May 2016. A total of 126 Staphylococcus aureus isolates from different clinical specimens like wound swabs, pus and urine received from inpatients and outpatients of this referral tertiary care hospital were included in the study. Staphylococci were obtained either as pure culture or as an isolate of a polymicrobial infection. These were incubated at $37^{\circ} \mathrm{C}$ for 24 hours. S. aureus was identified conventionally based on Gram's stain morphology, colony characteristics, and positive catalase and coagulase tests. The Staphylococcus species were identified on the basis of both slide and tube coagulase test. ${ }^{6} 100$ MRSA strains amongst the total 126 Staphylococcus aureus isolates were identified using cefoxitin disc (30 $\mu \mathrm{g})$ by Kirby-Bauer disc diffusion technique according Clinical and Laboratory Standards Institute (CLSI) guidelines 2014. ${ }^{7}$

All MRSA isolates were subjected to susceptibility testing by the Kirby-Bauer's disc diffusion method using different antimicrobial agents e.g., penicillin (10 units), clindamycin (2 $\mu \mathrm{g})$, ciprofloxacin (5 $\mu \mathrm{g})$, co-trimoxazole $(25 \mu \mathrm{g})$, azithromycin $(15 \mu \mathrm{g})$, gentamicin $(10 \mu \mathrm{g})$, linezolid $(30 \mu \mathrm{g})$, ofloxacin $(5 \mu \mathrm{g})$, tetracycline, amikacin, ampicillin, vancomycin $(30 \mu \mathrm{g})(\mathrm{Hi}-$ media). Zone diameter of the test strain was measured in millimetre with a template. All the MRSA isolates irrespective of their susceptibility pattern to Vancomycin by disc diffusion were resorted to E-test (bioMerieux) to determine the minimum inhibitory concentration (MIC) and confirmation of their resistance status. 0.5 McFarland equivalent inocula, prepared by using 18-24 hours old culture was inoculated on the Mueller-Hinton agar plates on which E-test strip was implanted according to the user guideline. Plates were incubated at $35^{\circ} \mathrm{C}$ for 24 hours before assessing the visible growth. The MIC is the value at which the elliptical zone of inhibition converges on the strips. Isolates with a vancomycin MIC $\leq 2 \mu \mathrm{g} / \mathrm{mL}$ were identified as Sensitive, 4 to $8 \mathrm{mg} / \mathrm{L}$ were identified as vancomycin-intermediate and isolates with a vancomycin MIC $>16 \mathrm{mg} / \mathrm{L}$ were identified as vancomycinresistant S. aureus (CLSI, 2014). ${ }^{7}$ Vancomycin breakpoints, according to which the current definition for VISA is an $S$. aureus isolate with a vancomycin broth MIC of $4-8 \mu \mathrm{g} / \mathrm{mL}$ since they use doubling dilutions of antibiotic (otherwise all isolates with MIC between 2 and $16 \mu \mathrm{g} / \mathrm{mL}$ are called VISA strains; strains with MIC $\leq 2 \mu \mathrm{g} / \mathrm{mL}$ are sensitive and $\geq 16 \mu \mathrm{g} / \mathrm{mL}$ are resistant). MIC >8 must be referred to a reference laboratory according to the CLSI guidelines. ${ }^{8}$ Quality control was performed using the $S$. aureus ATCC 25923 strain.

\section{Statistical Analysis}

The data collected were put into MS-Excel sheets for analysis. Percentages were used for interpretation of the data in this study.

\section{RESULTS}

The prevalence of MRSA isolates was (100/126) 79.36\%. Among 100 MRSA isolates, 5 strains were found to be resistant to vancomycin by disc diffusion test. Of these strains, only one isolate was found to be VISA showing an MIC range between 4$6 \mathrm{mg} / \mathrm{L}$, when tested by E-test (bioMerieux). And, amongst the 95 isolates which were sensitive by disc diffusion two more isolates were found to be Vancomycin Intermediate Staphylococcus aureus by E-strip method. Therefore, VISA isolates were observed in only $3 \%$ of isolates in our study.

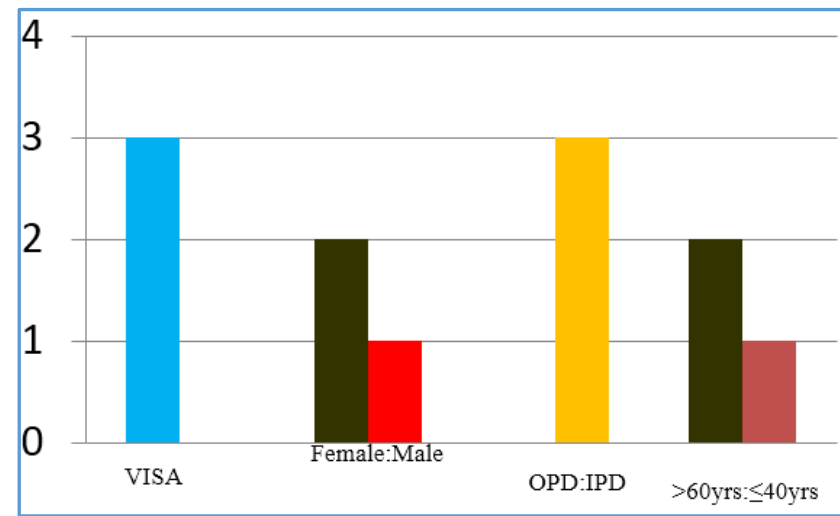

Figure 1. VISA According to Gender, IPD, OPD \& Age Groups

Figure 1 shows Vancomycin intermediate resistant Staphylococcus aureus according to gender, inpatient/outpatient department and age groups. In our study, the incidence of VISA was 3\%, out of which two were female and one was male. And all the VISA isolates were from inpatient department. Of the three VISA isolates, one was pus sample of a 40-year-old male patient from male surgery ward. And the other two VISA isolates were from urine samples, one from a 75-year-old female patient and another one from a 64year-old female patient from female medicine ward.

No Vancomycin resistant Staphylococcus aureus was detected.
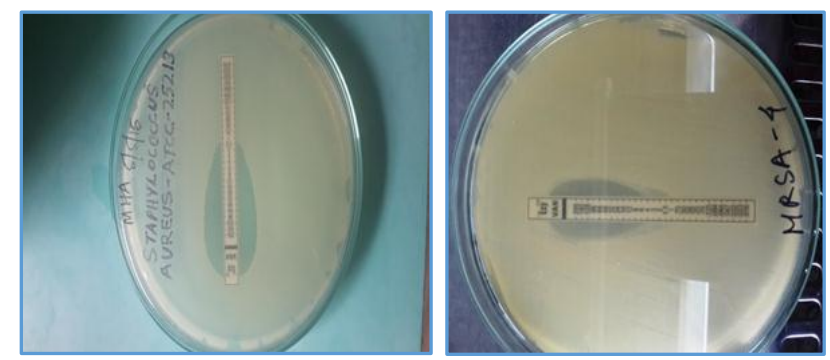

Figure 2. Showing MIC by E-strip Technique for (left) Staphylococcus Aureus 25213 and (right) MRSA Isolate.

Figure 2 (left) shows that the MIC of the control strain is about $1.5 \mathrm{mg} / \mathrm{L}$ which is $<2 \mathrm{mg} / \mathrm{L}$ and the picture on the right 
is of an MRSA isolate of a clinical sample which shows an MIC of $4 \mathrm{mg} / \mathrm{L}$.

\begin{tabular}{|c|c|}
\hline Antibiotic & Sensitivity (\%) \\
\hline Linezolid & 100 \\
\hline Vancomycin & 95 \\
\hline Ofloxacin & 79 \\
\hline *Nitrofurantoin & 76 \\
\hline Ciprofloxacin & 75 \\
\hline Gentamycin & 70 \\
\hline Tetracycline & 66 \\
\hline Amikacin & 62 \\
\hline Azithromycin & 60 \\
\hline **Clindamycin & 55 \\
\hline Cotrimoxazole & 24 \\
\hline Ampicillin & 10 \\
\hline Penicillin & 0 \\
\hline
\end{tabular}

Table 1. Showing Antibiotic Sensitivity Pattern amongst the MRSA isolates by Kirby Bauer's Disc Diffusion Method

*Nitrofurantoin only for urine isolates. ${ }^{* *}$ Clindamycin not for urine samples.

The antibiotic sensitivity pattern of MRSA isolates as shown in Table 1: linezolid (100\%), vancomycin (95\%), ofloxacin (79\%), nitrofurantoin (76\%), ciprofloxacin (75\%), gentamycin $(70 \%)$, tetracycline $(66 \%)$, amikacin $(62 \%)$, azithromycin (60\%), clindamycin (55\%), cotrimoxazole (24\%), ampicillin (10\%) and penicillin (0\%).

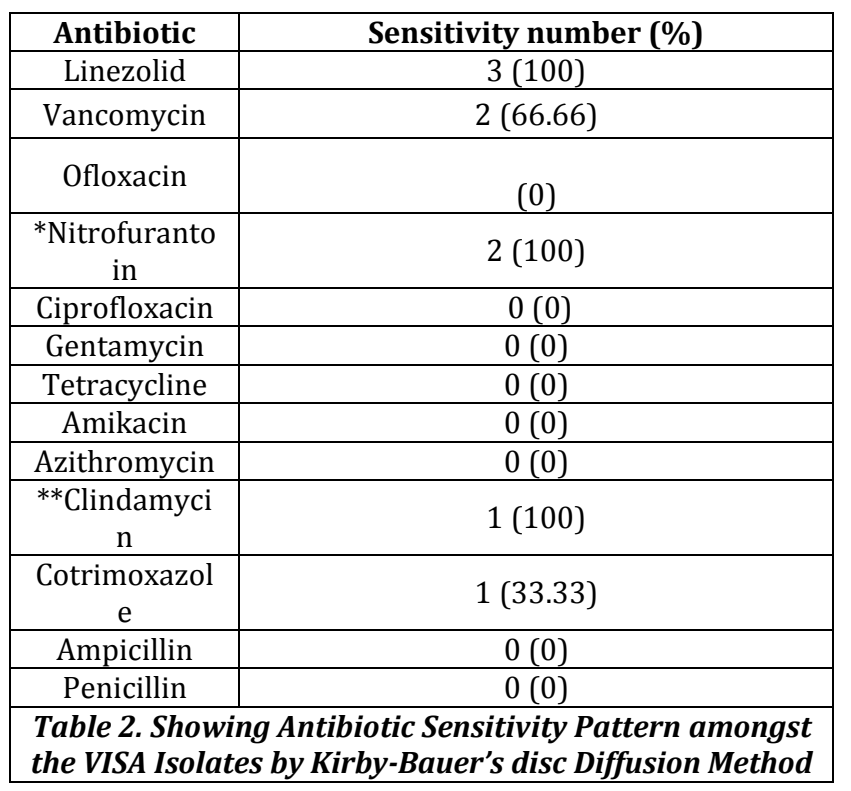

*Nitrofurantoin only for urine isolates.

**Clindamycin not for urine samples.

The antibiotic sensitivity pattern of the VISA isolates as shown in Table 2 linezolid (100\%), vancomycin (66.66\%), ofloxacin $(0 \%)$, nitrofurantoin $(100 \%)$, ciprofloxacin $(0 \%)$, gentamycin $(0 \%)$, tetracycline $(0 \%)$, amikacin $(0 \%)$, azithromycin (0\%), clindamycin $(100 \%)$, cotrimoxazole (33.33\%), ampicillin $(0 \%)$ and penicillin $(0 \%)$.

\section{DISCUSSION}

In our study, among 100 MRSA, 5 strains were found to be resistant to vancomycin by disc diffusion test. Of these strains, only one was found to be Vancomycin intermediate Staphylococcus aureus (VISA) by E-strip method. And, two more isolates amongst the 95 isolates which were sensitive by disc diffusion method, turned out to be VISA by E-strip method. As clearly stated in the CLSI guideline, vancomycin disc diffusion technique does not differentiate between vancomycin susceptible, intermediate and resistant strains. ${ }^{5}$ Therefore, a total of 3\% VISA was isolated in our study. Moses A et al found a higher incidence of $18.0 \%$ of VISA. ${ }^{9}$ Tiwari HK et al showed a prevalence of $1.8 \%$ VISA which is lesser in comparison to our study. ${ }^{10} \mathrm{Hu} \mathrm{H} \mathrm{C}$ et al also found a prevalence of $2.89 \%$ of VISA in a study conducted in Taiwan which is a comparable finding with our study. ${ }^{11}$

MRSA isolates were $100 \%$ resistant to Penicillin followed by Ampicillin (90\%) and Cotrimoxazole (76\%). 100\% sensitivity was seen with Linezolid followed by Ciprofloxacin (75\%), Ofloxacin (79\%), Nitrofurantoin (76\%), Amikacin (62\%), Gentamycin (70\%) and Tetracycline (66\%). B S Suresh et al also found that linezolid and ofloxacin were amongst the most sensitive drug for MRSA. ${ }^{11}$ The antibiotic sensitivity pattern was similar in cases of OPD and IPD isolates.

Amongst the VISA isolates, linezolid was the most sensitive for all the three isolates and nitrofurantoin was sensitive for both the urine isolates and clindamycin was also sensitive for the only pus isolate. ${ }^{12}$

In a study conducted by $\mathrm{K}$ Tandel et al, E-test was found to be a feasible alternative method for screening of VRSA as it is easy to perform and its cost effectiveness, but the study also found that E-test provides vancomycin MIC results consistently higher (by 0.5 to $2 \log 2$ dilutions) than those provided by reference agar dilution tests and so the study concluded by stating that Agar dilution test should be done on all those strains showing higher MIC (intermediate or resistant results) by E-test. In our study, we did not confirm the VISA isolates with agar dilution method. Therefore, there is a chance of the incidence of VISA isolates to be less than $3 \%$. But a study conducted by EH Natasha et al mentioned that a number of studies have shown that the E-test, MIC test seems to predict treatment failure better than broth microdilution testing, this may be a convenient and practical MIC determination method. 4

M Bilal Ahmad et al found that there is a difference between antibiogram of MRSA and MSSA isolates and therefore, routine E-testing of methicillin resistance should be done using cefoxitin disc which at present is the most sensitive method.13 In our study, we did not compare the antibiotic sensitivity pattern of the two groups MRSA and MSSA, which shall be done in our next study in near future.

In our study, isolation of the Van A/Van B genes by Polymerase Chain Reaction (PCR) was not done. However, in the study conducted by Tiwari and Sen, these genes were absent in the VISA and VRSA strains isolated even in the presence of phenotypic resistance to Vancomycin. Hence, the presence or absence of the resistant Van A/B genes does not necessarily rule out that strains are not VISA/VRSA. ${ }^{9}$ 


\section{Limitation}

Hetero-resistance vancomycin intermediate Staphylococcus aureus (hVISA) cases have been defined as those phenotypic isolates which on in-vitro susceptibility test is vancomycin susceptible (VSSA) but has a minority population of vancomycin intermediate (VISA). hVISA is responsible for vancomycin treatment failure but detection of hVISA was not done in our study.

\section{CONCLUSION}

This study emphasises the need to study the prevalence and antimicrobial susceptibility pattern of MRSA isolates areawise in order to guide policy on the appropriate use of antibiotics which would minimise the irrational use of vancomycin and so the emergence of resistance to vancomycin. So interpretation of susceptibility results should occur in discussion with relevant clinical personnel such as infectious-disease pharmacists and physicians, clinical microbiologists, and hospital antimicrobial-committee members.

\section{REFERENCES}

[1] Chaudhary M, Payasi A. Surveillance study for MRSA prevalence and susceptibility trends against mecA and vanA positive clinical isolates. International Journal of Advances in Pharmacy, Biology and Chemistry 2015;4(2):469-77.

[2] Singh HA, Aruna S. A study on the prevalence and antimicrobial susceptibility pattern of methicillin resistant Staphylococcus aureus in south Indian tertiary care hospital. International Journal of Current Research and Review 2014;6(23):14-7.

[3] Thati V, Shivannavar CT, Gaddad SM. Vancomycin resistance among methicillin resistant Staphylococcus aureus isolates from intensive care units of tertiary care hospitals in Hyderabad. Indian J Med Res 2011;134(5):704-8.

[4] Holmes NE, Johnson PD, Howden BP. Relationship between vancomycin-resistant Staphylococcus aureus, vancomycin-intermediate S. aureus, high vancomycin MIC, and outcome in serious S. aureus infections. Journal of Clinical Microbiology 2012;50(8):2548-52.
[5] Indian Network for Surveillance of Antimicrobial Resistance (INSAR) group, India. Methicillin resistant Staphylococcus aureus (MRSA) in India: prevalence \& susceptibility pattern. Indian Journal Medical Research 2013;137(2):363-9.

[6] Elmer K, Washington W, Staphen A, et al. Koneman's Color Atlas \& Textbook of diagnostic microbiology. $6^{\text {th }}$ edn. 2006:643-8.

[7] Clinical and Laboratory Standards Institute. Performance standards for antimicrobial susceptibility testing, 24th informational supplement (M100-S24). Wayne, Pa: Clinical and Laboratory Standards Institute, 2014.

[8] Tandel K, Praharaj AK, Kumar S. Differences in vancomycin MIC among MRSA isolates by agar dilution and $\mathrm{E}$ test method. Indian Journal of Medical Microbiology 2012;30(4):453-5.

[9] Moses A, Uchenna U, Nworie O. Epidemiology of vancomycin resistant Staphylococcus aureus among clinical isolates in a tertiary hospital in Abakaliki, Nigeria. American Journal of Epidemiology and Infectious Disease 2013;1(3):24-6.

[10] Tiwari HK, Sen MR. Emergence of vancomycin resistant Staphylococcus aureus (VRSA) from a tertiary care hospital from northern part of India. Biomed Central Infectious Diseases 2006;6:156.

[11] Hu HC, Kao KC, Chiu LC, et al. Clinical outcomes and molecular typing of heterogenous vancomycinintermediate Staphylococcus aureus bacteremia in patients in intensive care units. Biomed Central Infectious Diseases 2015;15:444.

[12] Sonth SB, Hadapad D, Gokale S, et al. Study of antimicrobial susceptibility pattern of methicillin resistant Staphylococcus aureus in a tertiary care hospital. International Journal of Current Microbiology and Applied Sciences 2015;4(10):924-7.

[13] MIR BA, Srikanth. Prevalence and antimicrobial susceptibility of methicillin resistant Staphylococcus aureus and coagulase-negative Staphylococci in a tertiary care hospital. Asian Journal of Pharmaceutical and Clinical Research 2013;6(3):231-4. 